\title{
Phase Retrieval through Focus Variation for Ultra-Resolution in Field-Emission Transmission Electron Microscopy
}

\author{
Wim Coene and Guido Janssen \\ Philips Research Laboratories, $5600 \mathrm{JA}$ Eindhoven, The Netherlands \\ Marc Op de Beeck and Dirk Van Dyck \\ EMAT, University of Antwerp (RUCA), B-2020 Antwerp, Belgium
}

(Received 30 July 1992)

\begin{abstract}
The use of a coherent field-emission electron source in transmission electron microscopy is combined with phase retrieval by digital processing of a focal image series. For the first time, a dramatic improvement of the high-resolution performance of the electron microscope beyond the usual "point-to-point" resolution has been realized: Experiments on a $200-\mathrm{kV}$ microscope with a point resolution of $0.24 \mathrm{~nm}$ reveal reconstructed information down to $0.14 \mathrm{~nm}$. Examples are shown in the field of high- $T_{c}$ superconductors and ferroelectric oxides. The oxygen sublattice in these structures is revealed.
\end{abstract}

PACS numbers: $61.16 . \mathrm{Di}$

For about 20 years now, high-resolution transmission electron microscopy (HRTEM) has proven its excellent capabilities for structure analysis down to the atomic scale for the study of lattice defects, interfaces, etc. [1,2]. In the run towards ultrahigh resolution in TEM [3], several recent instrumental improvements are promising: (1) reduction of spherical and chromatic aberration of the electron-optical lens system; (2) use of high voltages ( $\geq 1 \mathrm{MeV}$ ); and (3) use of a field-emission electron gun (FEG), with a much higher coherence than standard thermionic electron guns. In this Letter, we will focus on the FEG-TEM route towards ultrahigh resolution at intermediate voltages $(200-300 \mathrm{kV})$ using digital processing of focal image series.

The FEG has a higher brightness and lower energy spread than a thermionic source $\left(\beta=5 \times 10^{8}\right.$ and $1 \times 10^{6}$ $\mathrm{A} / \mathrm{cm}^{2} \mathrm{sr}, \Delta E=0.4$ and $0.8 \mathrm{eV}$, respectively). This yields a very good coherence for the FEG-TEM, and therefore its information limit is much better than the "point-topoint" resolution $\rho_{s}=0.64\left(C_{s} \lambda^{3}\right)^{0.25}$, which is only determined by spherical aberration $\left(C_{s}\right)$ and the electron wavelength $\lambda$. In traditional HRTEM, only the information down to $\rho_{s}$ is mostly considered to be meaningful. It can be interpreted directly for a thin specimen near optimum or Scherzer focus. However, in the gap between point and information resolution of the FEG-TEM, image interpretation is complicated by focusing effects and lens aberrations. Figure 1 shows a typical transfer function at Scherzer focus. The point resolution $\rho_{s}$ corresponds to the spatial frequency where the transfer function changes sign. Beyond $\rho_{s}$ for a FEG, the transfer function is rapidly oscillating, and the image is not easily interpretable. Going further underfocus, the oscillations in the transfer function shift to lower frequencies, and the overall oscillation speed is reduced. In principle, the interpretation problem can be solved indirectly by image simulation [1].

In HRTEM, it is both the phase and amplitude of the electron wave $(\phi)$ at the specimen's exit face that carry the true information about (electron scattering in) the specimen. On propagation from specimen to image plane, phase and amplitude are mixed up by the transfer function, which acts as a complex spatial frequency filter. Moreover, HRTEM image formation is a nonlinear process, in which it is the intensity $(I)$ at the image plane that is recorded, while the phase information is lost. In frequency space, the specimen-to-image interferences are given by

$$
\begin{aligned}
I(\mathbf{G})= & \phi(\mathbf{0}) \phi^{*}(-\mathbf{G}) T(\mathbf{0},-\mathbf{G})+\phi^{*}(\mathbf{0}) \phi(\mathbf{G}) T(\mathbf{G}, \mathbf{0}) \\
& +\int_{\mathbf{G}^{\prime} \neq \mathbf{0} ; \mathbf{G}^{\prime} \neq-\mathbf{G}} \phi\left(\mathbf{G}+\mathbf{G}^{\prime}\right) \phi^{*}\left(\mathbf{G}^{\prime}\right) T\left(\mathbf{G}+\mathbf{G}^{\prime}, \mathbf{G}^{\prime}\right) d \mathbf{G}^{\prime}
\end{aligned}
$$

with $\mathbf{G}(\mathbf{G} \neq \mathbf{0})$ the two-dimensional frequency vector. $T$ is the transmission cross coefficient (TCC) [4], which accounts for lens aberrations and coherence effects. The first and second terms in (1) represent the linear interfer-

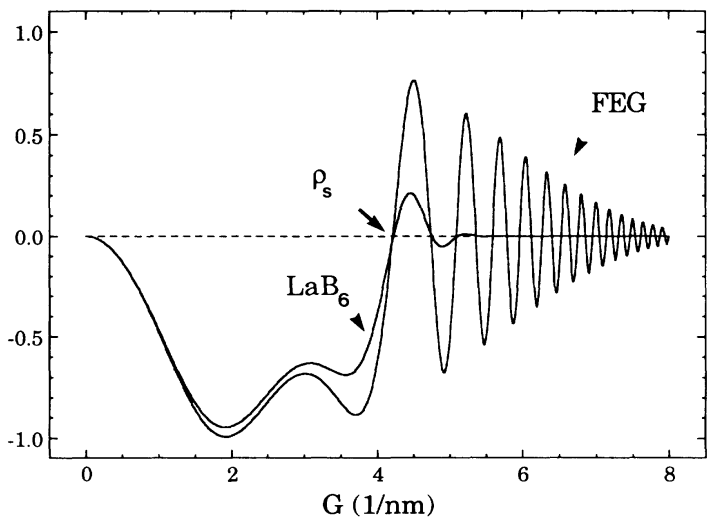

FIG. 1. Transfer functions (imaginary part) for a 200-kV microscope with $C_{s}=1.2 \mathrm{~mm}$ at Scherzer defocus $(-67 \mathrm{~nm})$, for a thermionic gun $\left(\mathrm{LaB}_{6}\right)$ and a FEG, as a function of spatial frequency $G$. 
ences between the transmitted electron beam $\phi(\mathbf{G}=\mathbf{0})$ and one of the diffracted electron beams $\phi(\mathbf{G} \neq \mathbf{0})$. Nonlinear interferences occur between two diffracted beams [third term in (1)]. "Holographic" methods are needed to retrieve the phase information, and to compensate for the transfer of the TEM. "Off-axis" electron holography is one possible route for phase retrieval [5]. We follow the route of "in-line" or "in-column" holography by direct reconstruction of phase and amplitude of $\phi$ out of a focal series of HRTEM images.

The idea of combining several focal images for increasing the information content is not new. The first proposals for reconstruction in the "linear imaging" regime date already from Schiske [6] and Saxton [7], leading to the early experimental results of Saxton [8,9]. Kirkland [10] was the first to tackle successfully nonlinear image reconstruction, matching the electron wave $\phi$ to the measured intensities $I$ by minimizing a least-squares functional, however, at the expense of an impractically huge computation time, even for few images of limited frame size. Recently, we have developed two workable solutions to circumvent these limitations. A first method aims at filtering out recursively the nonlinear contributions, so that high-speed linear reconstruction can be applied [11], i.e., for a focal series of $N$ images,

$$
\phi^{j+1}(\mathbf{G})=\sum_{n=1}^{N} F_{n}(\mathbf{G})\left[I_{n, \exp }(\mathbf{G})-I_{n, j}^{\mathrm{NL}}(\mathbf{G})\right],
$$

with $I_{n, \exp }$ the intensity of the $n$th experimental image, $I_{n, j}^{\mathrm{NL}}$ the nonlinear contributions for the $n$th image, estimated for $\phi^{j}$, and $F_{n}$ the $n$th filter function for linear reconstruction as given in [7]. Such a scheme is feasible for thin specimens with imaging conditions still close to linear. Second, we have devised a new variant of the Kirland reconstruction scheme within a maximum-likelihood (MAL) framework. A gain in computational efficiency with 3 to 4 orders of magnitude is realized, dependent on the image frame size. Full nonlinear MAL reconstruction of a focal series of 20 images of $512^{2}$ pixels requires now less than $1 \mathrm{~h}$ of CPU time on a 50-MFLOPS computer. The key to this improvement is an appropriate computational model, tailored to the coherence properties of the FEG. The very high brightness of the FEG implies very good spatial coherence. Temporal coherence, a combination of chromatic aberration and instabilities of voltage and current, is still the limiting factor, yielding effectively a focal averaging during recording of the HRTEM image. In our MAL algorithm, the focal averaging is built in explicitly, yielding for the intensity of the $n$th image in the focal series, in real space $(\mathbf{R})$

$$
I_{n}(\mathbf{R})=\int f_{\Delta}(\varepsilon) d \varepsilon\left|\mathrm{FT}_{\mathbf{G} \rightarrow \mathbf{R}}^{-1}\left[\phi(\mathbf{G}) t_{n, \varepsilon}(\mathbf{G})\right]\right|^{2} .
$$

$\mathrm{FT}^{-1}$ represents an inverse Fourier transform. $f_{\Delta}(\varepsilon)$ is the "chromatic" focal spread function, and $t_{n, \varepsilon}(\mathbf{G})$ the effective transfer function, i.e., the product of the pure phase transfer function and the envelope factor for spatial coherence:

$t_{n, \varepsilon}(\mathbf{G})=\exp \left\{-2 \pi i \chi_{n, \varepsilon}(\mathbf{G})\right\} \exp \left\{-\left(\frac{\pi \alpha}{\lambda}\right)^{2}\left[\nabla \chi_{n, \varepsilon}(\mathbf{G})\right]^{2}\right\}$.

$\alpha$ is the half-angle of beam convergence, and $\chi_{n, \varepsilon}(\mathbf{G})$ and $\nabla \chi_{n, \varepsilon}(\mathbf{G})$ the wave aberration function and its spatial gradient, with the former given by

$$
\chi_{n, \varepsilon}(\mathbf{G})=\frac{1}{2}\left(\Delta f_{n}+\varepsilon\right) \lambda G^{2}+\frac{1}{4} C_{s} \lambda^{3} G^{4} .
$$

$\Delta f_{n}$ is the focus of the $n$th HRTEM image. Using $t_{n, \varepsilon}(\mathbf{G})$ implies a factorized approach for the spatial coherence envelope factor in the TCC [in (1)]. This is only allowed for low values of $\alpha\left(\leq 5 \times 10^{-5} \mathrm{rad}\right)$ as can be achieved with a FEG. For the iteration cycle $j \rightarrow j+1$ for the specimen wave $\phi$ in the recursive MAL method, we obtain, using a focal series of $N$ images with transfer functions $t_{n, \varepsilon}(\mathbf{G}), n=1, \ldots, N$,

$$
\left.\phi^{j+1}(\mathbf{G})=\phi^{j}(\mathbf{G})+\frac{\gamma}{N} \sum_{n=1}^{N} \int f_{\Delta}(\varepsilon) d \varepsilon t_{n, \varepsilon}^{*}(\mathbf{G}) \mathrm{FT}_{\mathbf{R} \rightarrow \mathbf{G}}\left[I_{n, \exp }(\mathbf{R})-I_{n, j}(\mathbf{R})\right] \mathrm{FT}_{\mathbf{G} \rightarrow \mathbf{R}}^{-1}\left[\phi^{j}(\mathbf{G}) t_{n, \varepsilon}(\mathbf{G})\right]\right],
$$

with $I_{n, j}$ the estimated intensity for $\phi^{j}$, and $\gamma$ a convergence parameter. Relations (3) and (6) enable us to benefit from fast Fourier transforms, thus avoiding weighted correlations in frequency space for the propagation between specimen and image plane [as in (1)].

Apart from the FEG with its coherence and the design of a proper computational model, several other developments have been essential for practical image reconstruction: (i) slow-scan CCD cameras for TEM [12] enabling linear and direct digital image acquisition; (ii) computer control of the TEM; (iii) high-speed computers $(>50$ MFLOPS); and (iv) accurate measurement of electronoptical parameters [13].

We report here on two experiments of phase retrieval using a Philips CM20 FEG-TEM at $200 \mathrm{kV}(\lambda=2.5 \mathrm{pm}$,
$C_{s}=1.2 \mathrm{~mm}, \rho_{s}=0.24 \mathrm{~nm}$ ). The first experiment is performed on $\mathrm{YBa}_{2} \mathrm{Cu}_{4} \mathrm{O}_{8}$ in [100] orientation. With conventional HRTEM using a thermionic gun on the structurally related $1: 2: 3$ compound, the positions of the metal atoms can be determined directly [14], but the resolution at $200 \mathrm{kV}$ is not sufficient to visualize the light oxygens. At $400 \mathrm{kV}$, imaging of the oxygen sublattice becomes more feasible [15], but the experimental effects are weak and not directly obvious, and need to be supported by image simulation [16,17]. Moreover, HRTEM at $400 \mathrm{kV}$ has the disadvantage of increased radiation damage. The structure of $\mathrm{YBa}_{2} \mathrm{Cu}_{4} \mathrm{O}_{8}$, shown in Fig. 2, has an extra $\mathrm{CuO}$ plane compared to the $1: 2: 3$ compound. Figure 3 shows a few images of the focal series recorded with the 


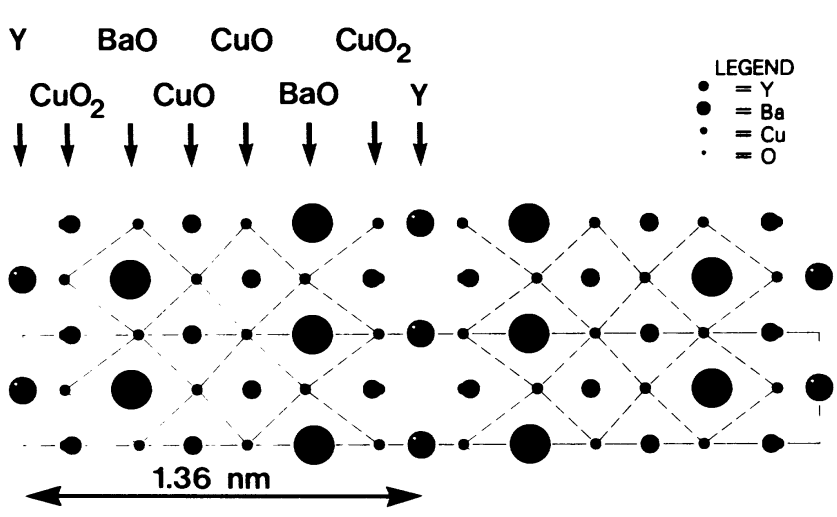

FIG. 2. Structure model of $\mathrm{YBa}_{2} \mathrm{Cu}_{4} \mathrm{O}_{8}$ viewed along [100]. The parameters of the unit cell in projection are $b=0.387 \mathrm{~nm}$, $c=2.719 \mathrm{~nm}$.

200-kV FEG-TEM. The focus ranges from just below Scherzer focus $(\sim-67 \mathrm{~nm})$ to further underfocus, clearly revealing different contrasts. The relevant information about the structure is scrambled over the whole focal series in a complicated way, severely frustrating image interpretation. The configuration of the heavy atoms $\mathrm{Ba}$, $\mathrm{Y}$, and the $\mathrm{Cu}$ atoms in the $\mathrm{CuO}_{2}$ planes can be seen as prominent white dots at the focus of $-91 \mathrm{~nm}$. There, faint crosses represent the information about the $\mathrm{CuO}$ planes, the configuration of which is better observable at the larger underfocus of $-271 \mathrm{~nm}$. A zigzag pattern of white dots representing both successive $\mathrm{CuO}$ planes is observed at the foci -62 and $-206 \mathrm{~nm}$. Contrast reversals occur between different focus conditions (e.g., -62 $\mathrm{nm}$ vs $-91 \mathrm{~nm}$ ), and at some defoci, the image is not a simple white dot pattern, but rather a pattern of crosses and broader patches of intensity $(-77 \mathrm{~nm})$. Figure 4 shows the reconstructed phase which, since this specimen is very thin $(\sim 3 \mathrm{~nm})$, is proportional to the electrostatic potential projected along the direction of electron incidence, and therefore it yields a good representation of the projected crystal structure. All atom types are revealed in this single "image," and brightness, i.e., value of reconstructed phase, indicates directly the relative mass. The oxygen stacks in the $\mathrm{BaO}$ and $\mathrm{CuO}$ planes are clearly resolved, with the oxygens represented by white dots between more pronounced white dots that indicate the heavy atoms $(\mathrm{Cu}, \mathrm{Ba})$. The smallest $\mathrm{Cu}-\mathrm{O}$ distances in [100] projection are about $0.18 \mathrm{~nm}$. The $\mathrm{Y}$ atoms show up as the largest white dots; in the neighboring $\mathrm{CuO}_{2}$ planes the $\mathrm{Cu}$ atoms are clearly seen. Even the oxygens in the O-Y-O stacks ( $O-Y$ distance $=0.14 \mathrm{~nm}$ ) are observed as faint white dots, especially clear in the thicker specimen areas, due to the increased effect of multiple electron scattering.

The second experiment is carried out on the ferroelectric oxide $\mathrm{Ba}_{2} \mathrm{NaNb}_{5} \mathrm{O}_{15}$ in [001] orientation. The $\mathrm{Nb}$ $\mathrm{Ba}$ and $\mathrm{Na}-\mathrm{O}$ sublattices are shown in the inset of Fig. 5. HRTEM at $200 \mathrm{kV}$ with a thermionic source [18] has

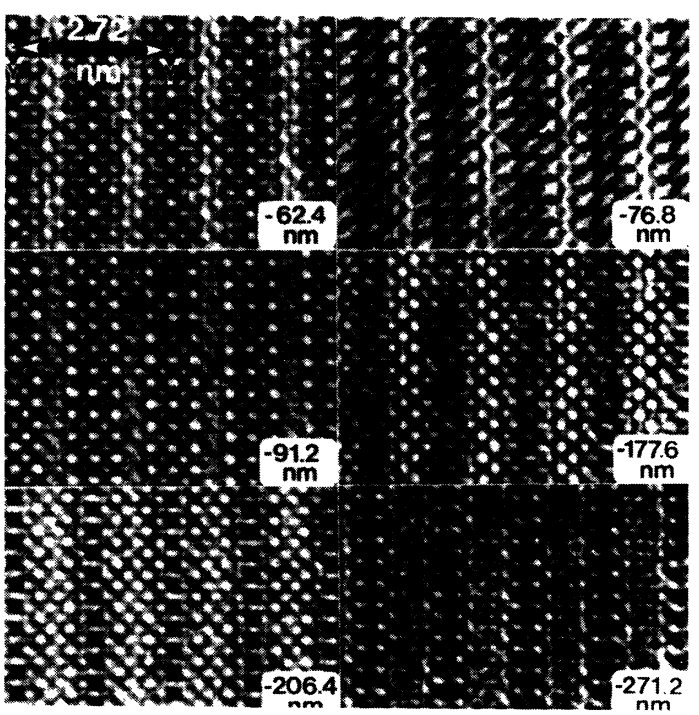

FIG. 3. HRTEM images of a focal series recorded from $\mathrm{YBa}_{2} \mathrm{Cu}_{4} \mathrm{O}_{8}$ in [100] orientation. The focus settings are indicated.

shown that the sublattices of the three metal atoms can be selectively imaged by properly changing focus; the oxygen sublattice could not be revealed. The results of phase retrieval with the 200-kV FEG-TEM are shown in Fig. 5. The reconstructed amplitude reveals the heavy atoms $(\mathrm{Nb}, \mathrm{Ba})$. The light atoms $(\mathrm{O}, \mathrm{Na}, \mathrm{O}-\mathrm{Na}$ distance $<0.19 \mathrm{~nm}$ ), are retrieved in the reconstructed phase as a stacking of $\mathrm{O}$ triangles and $\mathrm{O}$ squares, the latter having a $\mathrm{Na}$ atom in the center. Amplitude and phase yield complementary information, because the specimen is thicker $(\sim 10 \mathrm{~nm})$ and cannot be regarded as a phase object.

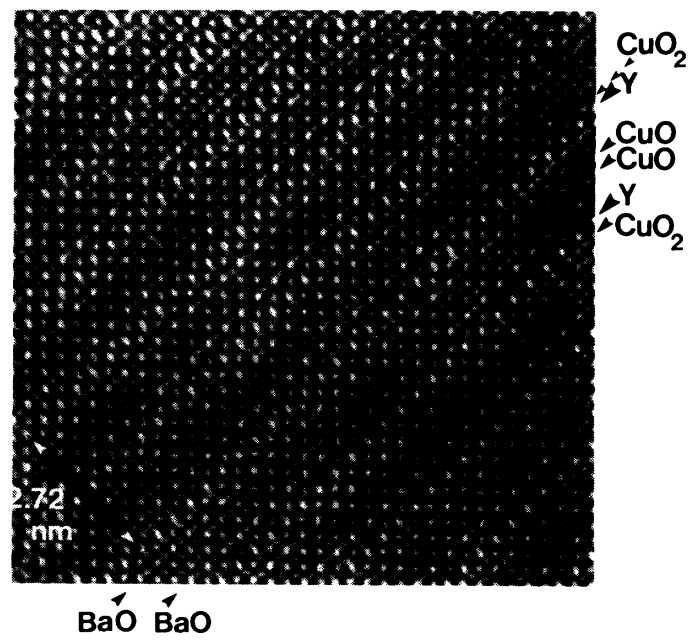

FIG. 4. Reconstructed phase from [100]-oriented $\mathrm{YBa}_{2}-$ $\mathrm{Cu}_{4} \mathrm{O}_{8}$. Specimen thickness increases from left bottom to top right. 


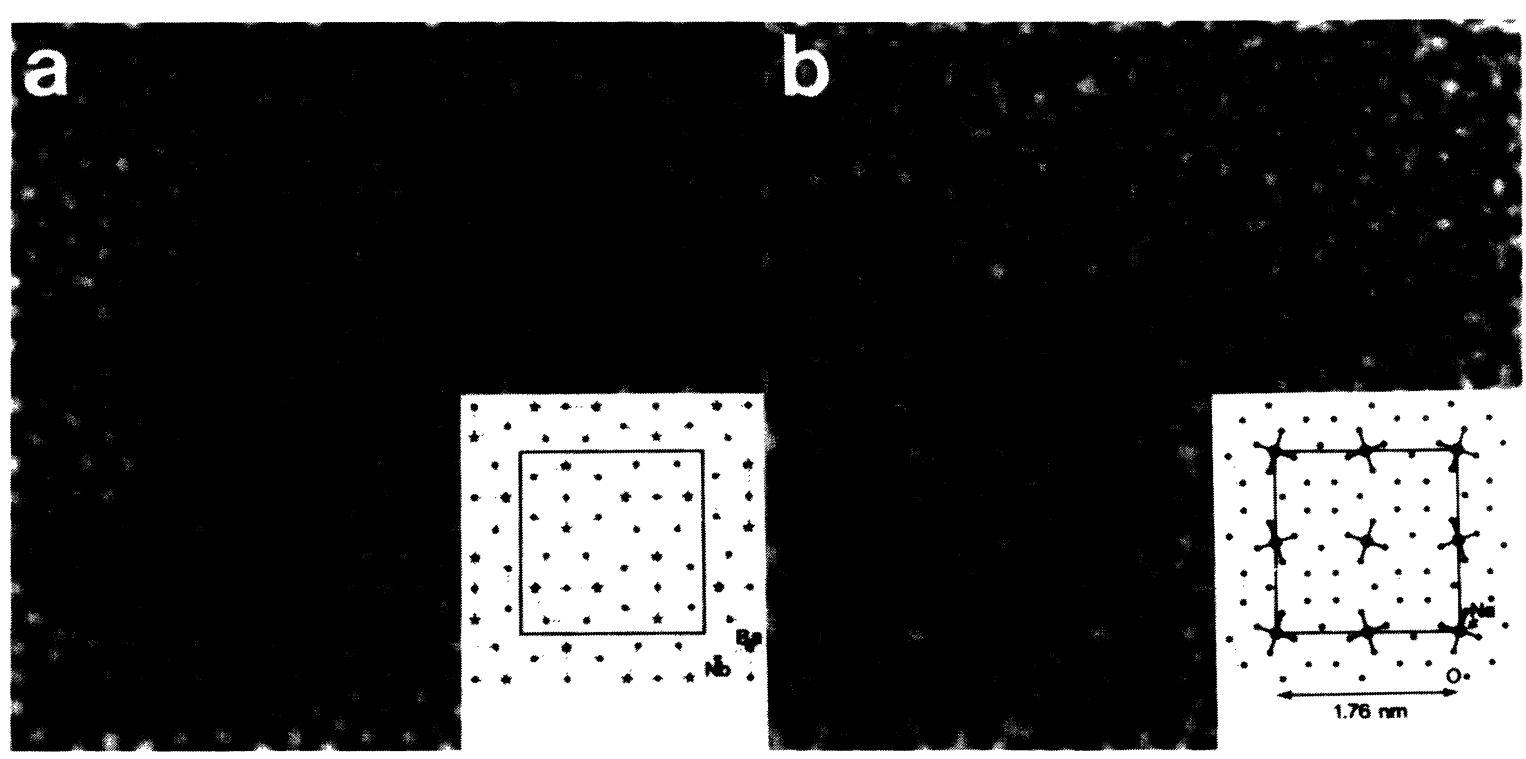

FIG. 5. Reconstructed amplitude (a) and phase (b) from [001]-oriented $\mathrm{Ba}_{2} \mathrm{NaNb}_{5} \mathrm{O}_{15}$ ( $\mathrm{Ba}-\mathrm{Nb}$ and $\mathrm{Na}-\mathrm{O}$ sublattices of structure in inset; those $\mathrm{O}$ atoms, coincident with the $\mathrm{Nb}$ atoms in this projection, are omitted; unit cell parameters are $a=1.759 \mathrm{~nm}, b=1.762$ $\mathrm{nm}$, and $c=0.400 \mathrm{~nm}$ ).

The experiments reported here demonstrate the striking improvement in resolution that is obtained with digital image reconstruction on a FEG-TEM at $200 \mathrm{kV}$. Although the point resolution is only $0.24 \mathrm{~nm}$, information even down to $0.14 \mathrm{~nm}$ is retrieved in the reconstructed image. Moreover, one has to consider the large differences in atomic mass. $\mathrm{Y}, \mathrm{Ba}, \mathrm{Cu}$, and $\mathrm{Nb}$ are heavy atoms, and will scatter the incident electrons strongly. O and $\mathrm{Na}$ are light and weakly scattering atoms, and will contribute only moderately to the HRTEM images. As a result of the coherence of the FEG, the information from the light-atom sublattice survives into the HRTEM images and is faithfully unraveled by image reconstruction.

This work is part of the BRITE-EURAM program nr. 3322 of the European Community. M. Op de Beeck is indebted to the Belgian IWONL for financial support. Dr. T. Krekels is acknowledged for joining the experiment on his $\mathrm{Y}-\mathrm{Ba}-\mathrm{Cu}-\mathrm{O}$ sample. We thank Professor G. $\mathrm{V}$ an Tendeloo for providing the $\mathrm{Ba}-\mathrm{Na}-\mathrm{Nb}-\mathrm{O}$ crystal.

[1] J. C. H. Spence, Experimental High Resolution Electron Microscopy (Oxford Univ. Press, Oxford, 1988).

[2] C. W. T. Bulle-Lieuwma, W. Coene, and A. F. de Jong, Adv. Mater. 3, 368 (1991).

[3] J. M. Cowley, in Proceedings of the 49th EMSA '91 (San Francisco Press, California, 1991), p. 650.

[4] K. Ishizuka, Ultramicroscopy 5, 55 (1980).
[5] H. Lichte, Adv. Opt. Electron Microsc. 12, 25 (1991).

[6] P. Schiske, in Image Processing and Computer aided Design in Electron Optics (Academic, New York, 1973), p. 82.

[7] W. O. Saxton, in Computer Techniques for Image Processing in Electron Microscopy (Academic, New York, 1978).

[8] W. O. Saxton, J. Microsc. Spectrosc. Electron. 5, 661 (1980).

[9] W. O. Saxton, in Proceedings of the Eleventh ICEM (Kyoto), post deadline paper 1, 1986 (unpublished).

[10] E. J. Kirkland, Ultramicroscopy 15, 151 (1984).

[11] D. Van Dyck and M. Op de Beeck, in Proceedings of the Twelfth ICEM, Seattle (San Francisco Press, California, 1990), p. 26.

[12] I. Daberkow, K.-H. Herrmann, L. Liu, and W. D. Rau, Ultramicroscopy 38, 215 (1991).

[13] A. F. de Jong and A. J. Koster, in Scanning Microscopy, Proceedings of the Tenth Pfefferkorn Conference on Image and Signal Processing in Electron Microscopy, Cambridge, 1991 (to be published).

[14] Y. Yan and M. G. Blanchin, Philos. Mag. A 61, 513 (1990).

[15] Y. Yan and M. G. Blanchin, Phys. Rev. B 43, 13717 (1991).

[16] A. Ourmazd and J. C. H. Spence, Nature (London) 329, 425 (1987)

[17] W. Krakow and T. M. Shaw, J. Electron Microsc. Tech. 8, 273 (1988).

[18] G. Van Tendeloo, D. Van Dyck, and S. Amelinckx, Ultramicroscopy 19, 235 (1986). 


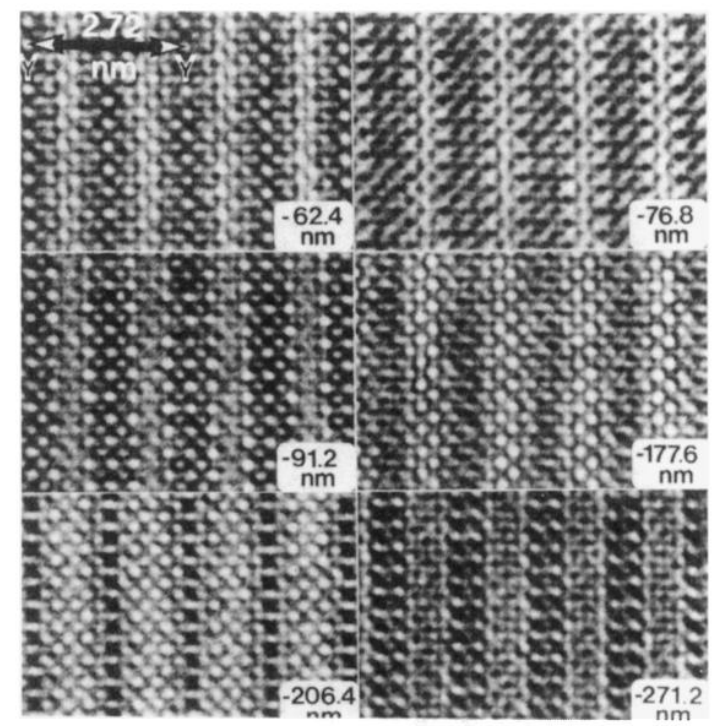

FIG. 3. HRTEM images of a focal series recorded from $\mathrm{YBa}_{2} \mathrm{Cu}_{4} \mathrm{O}_{8}$ in [100] orientation. The focus settings are indicated. 


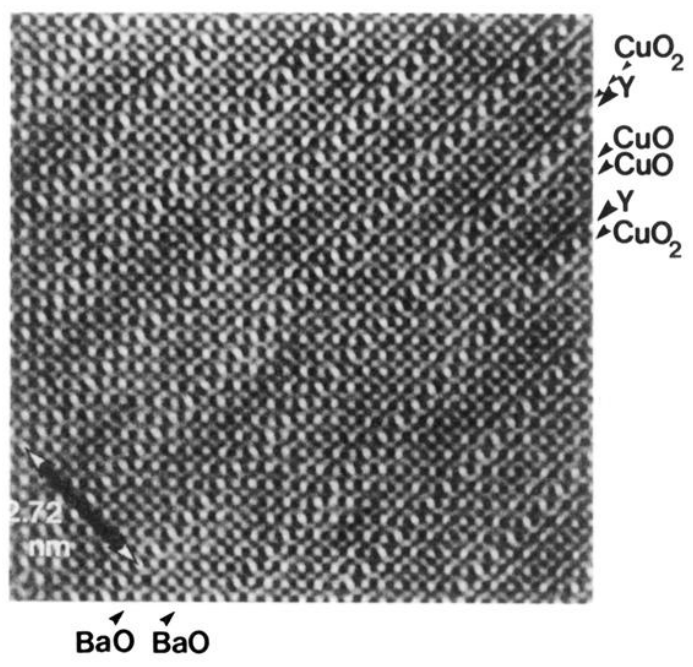

FIG. 4. Reconstructed phase from [100]-oriented $\mathrm{YBa}_{2-}$ $\mathrm{Cu}_{4} \mathrm{O}_{8}$. Specimen thickness increases from left bottom to top right. 


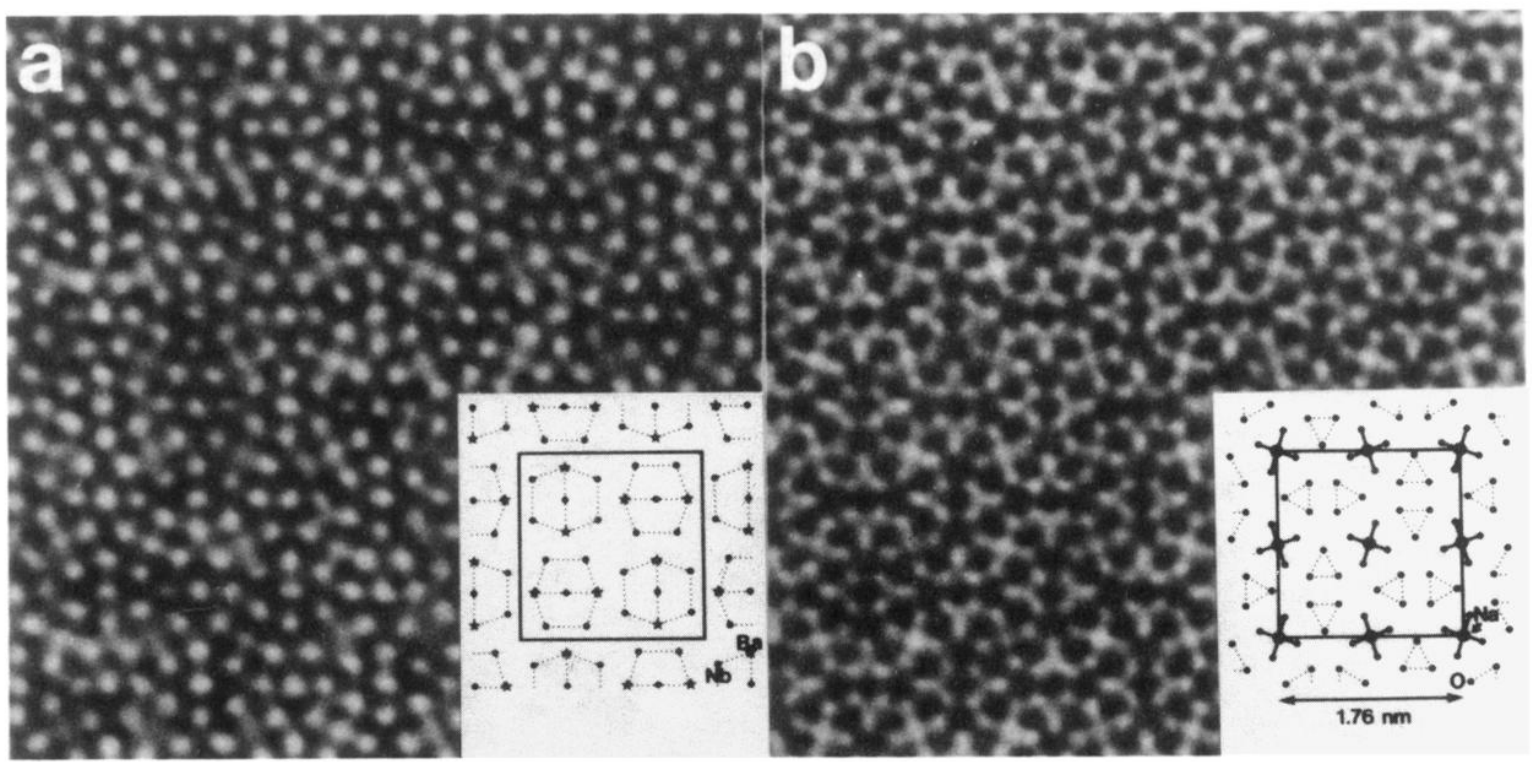

FIG. 5. Reconstructed amplitude (a) and phase (b) from [001]-oriented $\mathrm{Ba}_{2} \mathrm{NaNb}_{5} \mathrm{O}_{15}$ ( $\mathrm{Ba}-\mathrm{Nb}$ and $\mathrm{Na}-\mathrm{O}$ sublattices of structure in inset; those $\mathrm{O}$ atoms, coincident with the $\mathrm{Nb}$ atoms in this projection, are omitted; unit cell parameters are $a=1.759 \mathrm{~nm}, b=1.762$ $\mathrm{nm}$, and $c=0.400 \mathrm{~nm}$ ). 